\title{
System Design and Performance of the Two-Gyro Science Mode For the Hubble Space Telescope
}

\author{
Michael Prior \\ NASA Goddard Space Flight Center \\ Larry Dunham \\ Jackson and Tull \\ To be presented at the \\ $56^{\text {th }}$ International Astronautical Conference, Fukuoka Japan, October 17-21, 2005.
}

\section{Abstract}

For fifteen years, the science mission of the Hubble Space Telescope (HST) required using at least three of the six on-board rate gyros for attitude control. Failed gyros were eventually replaced through Space Shuttle Servicing Missions. The tragic loss of the Space Shutle Columbia has resulted in the cancellation of all planned Shuttle based missions to HST. While a robotic servicing mission is currently being planned instead, controlling with alternate sensors to replace failed gyros can extend the HST science gathering until a servicing mission can be performed, and also extend science at HST's end of life. Additionally, sufficient performance may allow a permanent transition to operations with less than 3 gyros (by intentionally turning off working gyros saving them for later use) allowing for an even greater science mission extension. To meet this need, a Two Gyro Science (TGS) mode has been designed and implemented using magnetometers (Magnetic Sensing System - MSS), Fixed Head Star Trackers (FHSTs), and Fine Guidance Sensors (FGSs) to control vehicle rate about the missing gyro input axis.

The development of the TGS capability is the largest re-design of HST operations undertaken, since it affects several major spacecraft subsystems, the most heavily being the Pointing Control System (PCS) and Flight Software (FSW). Additionally, and equally important, are the extensive modifications and enhancements of the Planning and Scheduling system which must now be capable of scheduling science observations while taking into account several new constraints imposed by the TGS operational modes (such as FHST availability and magnetic field geometry) that will impact science gathering efficiency and target availability. This paper discusses the systems engineering design, development, and performance of the TGS mode, now in its final stages of completion. 


\title{
SYSTEM DESIGN AND PERFORMANCE OF THE TWO-GYRO SCIENCE MODE FOR THE HUBBLE SPACE TELESCOPE
}

\author{
Michael Prior \\ Hubble Space Telescope Project Deputy Operations Manager (Acting) \\ NASA Goddard Space Flight Center \\ Greenbelt, Maryland, USA \\ mprior@hst.nasa.gov \\ Larry Dunham \\ Hubble Space Telescope Operations Systems Engineering Manager \\ Vantage Systems Inc. \\ Lanham, Maryland, USA \\ ldunham@hst.nasa.gov
}

\begin{abstract}
For fifteen years, the science mission of the Hubble Space Telescope (HST) required using three of the six on-board rate gyros for attitude control. Failed gyros were eventually replaced through Space Shuttle Servicing Missions. To ensure the maximum science mission life, a Two Gyro Science (TGS) mode has been designed and implemented with performance comparable to 3-gyro operations. The excellent performance has enabled a transition to operations with 2 gyros (by intentionally turning off a running gyro to save it for later use), and allows for an even greater science mission extension. Predictions show the gain in mission life approaching two years. In TGS mode, the rate information formerly provided by the third gyro is provided by another sensor. There are three submodes, each defined by the sensor used to provide the missing rate information. The three sensor suites are the magnetometers (Magnetic Sensing System - MSS), Fixed Head Star Trackers (FHSTs), and Fine Guidance Sensors (FGSs). Although each sensor suite has limitations, when used sequentially they provide the means to transition from relatively large, post-maneuver attitude errors of up to ten degrees, to the arcsecond errors needed to transition to fine pointing required for science observing. Once observing, boresight jitter is in the range of 8-10 milli-arcseconds versus 3-4 milli-arcseconds using 3 gyros. At these low jitter levels scientists see no degradation in image quality in TGS mode.
\end{abstract}

\section{INTRODUCTION}

The effort to develop a Two Gyro Science (TGS) capability began with a feasibility study in May of 2003 , after the loss of two gyros and several delays to the Servicing Mission 4 launch schedule This substantially increased the risk of having less than three functioning gyros remaining prior to the Servicing Mission. Previously, this risk was realized just prior to Servicing Mission 3A in 1999. when science operations halted for $\sim 1.5$ months until a new suite of gyros was installed 1 . The HST Project thus embarked upon the TGS development effort to avoid another science hiatus. At that time, expectations were that the TGS capability would only be utilized as a contingency mode (i.e., when only two gyros remained) due to the estimates of

\footnotetext{
${ }^{1}$ In the normal mode of operations at least 3 gyros are needed in the control loop. An option exists to use 4 gyros that allows for autonomous continued operations in the event of any single gyro failure. The HST Project had proactively gone from a default of 4 gyros in the control loop to 3 gyros in 2001. This had no impact on performance but caused gyro anomalies to now result in a temporary halt in science.
} 
overall system level performance that could be achieved-- mostly in the area of pointing jitter.

With the January 2004 cancellation of Servicing Mission 4, it became certain that the TGS capability would be utilized under the original contingency plan. However, successful on-orbit testing has shown that it produces science of the same quality as the original three-gyro control mode, with relatively minor impacts to overall science yield. With this realization, and quantifiable and acceptable impacts to science scheduling, the TGS capability is being utilized proactively as a normal operations mode to maximize the HST's lifetime.

\section{LIFE EXTENSION POTENTIAL}

Since the gyros have been and still are the limiting component, then just how much additional overall observatory lifetime can a TGS mode provide? The answer must include the evaluation of several factors including: 1) individual gyro run-time histories and construction, 2) history of gyro failures over the life of the mission, 3) the sequence of gyro pairs that are used, and 4) the risk and impact of a gyro not restarting.

Individual gyro lifetime estimates were determined from reliability models based upon Weibull probability analysis. Difference among the gyro run-time histories (i.e. how many hours a gyro has been used), and minor differences in construction were factored into the analysis ${ }^{2}$.

Taking these factors into account in the overall reliability model result in varying estimates for observatory lifetime based mostly upon how the gyros are used (assuming no other spacecraft component failures). Given the four healthy gyros

\footnotetext{
${ }^{2}$ All but one of the current on-orbit gyros was built using an improved fluid and fluid fill method that potentially reduced both corrosion and contamination. Failure of the flex leads due to corrosion and failure followed by rotor restriction due to contamination in the suspension fluid have been the major causes of gyro failure. Failures due to each root cause have been nearly equal in number.
}

available in mid-2005, different pair combinations that are chosen for initial use, and after subsequent failures, produce different total end-to-end lifetime estimates. Ultimately, to exploit the potential increased lifetime to the fullest requires a choice of gyro pair sequencing that maximizes mission lifetime in two simultaneous cases: 1) a turned off gyro restarts, and 2) a turned off gyro does not restart $^{3}$. In other words, picking the pair sequencing that maximize the best-case lifetime while simultaneously minimizing the penalty of a failed restart. The result is a best-case lifetime gain of 21 months beyond a purely 3 -gyro mode of operation and 13 months in the case a turned off gyro fails to restart ${ }^{4}$.

\section{OPERATIONS AND TGS MODES}

The HST was originally designed to use at least three gyros as the primary sensors for vehicle attitude control. The utilization of 3 gyros provides precise and accurate rate control at all times, allowing even large vehicle slews to be performed with resulting attitude errors that rarely exceed $\sim 100$ arcseconds. Typically, after every slew greater than 30 degrees (or when the projected attitude error exceeds 70 arcseconds), onboard attitude updates using Fixed Head Star Tracker (FHST) ${ }^{5}$ data are performed to reduce errors within a Fine Guidance Sensor (FGS) search radius of $\sim 50$ arcsec. Each of the three FGSs ${ }^{6}$ contains dual axis interferometers used to provide precise measurement of guide star positions and are used

\footnotetext{
Although the risk of a failure to testant is shown to be small based upon combined ground based and on-orbit experience (hundreds of restarts have been performed in ground based testing and tens of restarts have been performed on-orbit) it must be accounted for in the expected lifetime estimates and risk planning.

4 Assumes proactive use of TGS mode starting in August 2005 .

${ }^{5}$ There are 3 FHSTs, each with an $8^{\circ} \times 8^{\circ}$ field of view, oriented away from the telescoped boresight.

${ }^{6}$ Each FGS has a 4 arcminute $x 90^{\circ}$ arc field of view aligned with the telescope boresight. Typically, 2 FGSs are used to guiding while in 3-gyro normal mode with an option to guide using one FGS. In TGS mode 2 FGSs must be used for guiding.
} 
with gyro data to hold spacecraft attitude during science gathering. They can spiral search up to 120 arcseconds to find stars between $9^{\text {th }}$ and 14.5 visual magnitude. The Magnetic Sensing System (MSS), consisting of two magnetometers, is used primarily for momentum management and does not directly provide input to the attitude control portion of the Pointing Control System (PCS).

In TGS mode, the rate information that was formerly provided by the third gyro must now be provided by another sensor. The two remaining gyros are capable of sensing rate about a plane formed by their input axes but not about an axis normal to the plane $-\mathrm{G}_{\mathrm{x}}$ (Figure 1 ). This naturally leads to three submodes, each defined by the sensor used to provide the missing rate information about $\mathrm{G}_{\mathrm{x}}$. The three sensors that are utilized are the MSS, FHSTs, and FGSs, all of which have limitations, but when used together can provide the means to transition from relatively large attitude errors following a slew to the small errors needed to transition to fine pointing using the FGSs and the gathering of science.

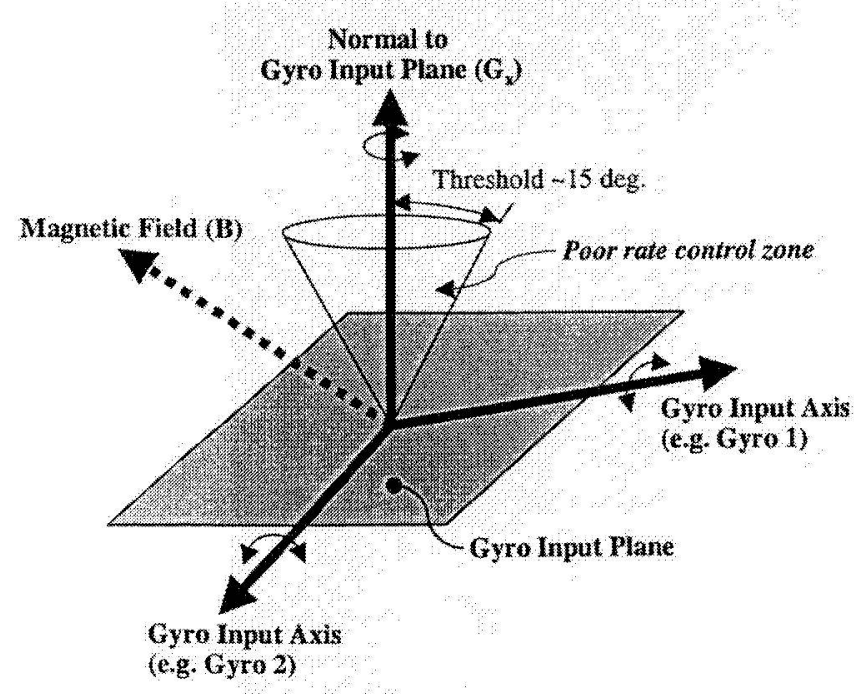

Figure 1. Gyro plane and $\mathrm{Gx}$ axis geometry definition. Gyro input axes are generally not orthogonal.

3.1. Magnetometer Sensing System/Two Gyro mode (M2G)
The first submode is the Magnetometer Sensing System/Two Gyro mode (M2G). M2G compares the MSS data (measuring the Earth's magnetic field vector) to the on-board magnetic field model while measuring the rate of change of the orientation of the field to provide both spacecraft body rate and attitude control. Since the MSS data is always available (i.e. there are no occultations due to sensor field of view restrictions, South Atlantic Anomaly passages, etc.), M2G is used for all large slews and as the fall back, lowest level mode in the case of FHST and/or FGS occultations. $\mathrm{M} 2 \mathrm{G}$ nominal attitude error is $\sim 1^{\circ}$, but can be as high as $10^{\circ}$. Such large attitude errors are a result of both the inherent noise in the measurement of the magnetic field vector, as well as occasional unfavorable geometric alignment between the field and the normal to the gyro input plane. Field orientations within $15^{\circ}$ of the $\mathrm{G}_{\mathrm{x}}$ axis, combined with a slow moving field produce the worst case errors (Figure 1). The rate error is nominally 0.01 degrees/second but can be as high as 0.03 degrees/second during the unfavorable alignments. The $\mathrm{M} 2 \mathrm{G}$ mode requirement is that the attitude error be within the capability of the FHST/Two Gyro mode (T2G) attitude determination and correction capability limit of 10 degrees.

\subsection{Fixed Head Star Tracker/Two Gyro mode (T2G)}

The second submode is the Fixed Head Star Tracker/Two Gyro mode (T2G). T2G is used for rate damping and attitude determination and correction, allowing the large body rates and attitude errors present in $\mathrm{M} 2 \mathrm{G}$ to be sufficiently reduced to permit the next higher submode (FGS/Two Gyro mode) to be entered where science observations are performed. It is thus a transition mode that allows spanning the loose attitude control of M2G to the very precise and accurate control of the highest mode. To accomplish this the set of three FHSTs are used extensively in a sequence of operations that begins by first controlling spacecraft body rates using one of the FHSTs. This is followed by an On-Board Attitude 
Determination and correction (OBAD) consisting of a star mapping operation requiring the use of two trackers simultaneously, followed by an onboard FSW-based attitude determination (utilizing the star data) and an attitude correction slew. The $O B A D$ is repeated a second time to further reduce attitude errors. Since spacecraft body rates are controlled throughout the $\mathrm{T} 2 \mathrm{G}$ period, continuous FHST visibility by the three FHSTs as a set (for rate control) is necessary. Additionally, OBAD execution requires two distinct periods where continuous and simultaneous visibility by two trackers is necessary.

The requirement for $\mathrm{T} 2 \mathrm{G}$ is that the attitude error be brought well within a 90 aresecond FGS guide star search radius after the second OBAD. High fidelity simulations and on-orbit test data demonstrate that even with initial attitude errors as high as 10 degrees, attitude determination and correction are successful in reducing final attitude error to within the required tolerance.

\subsection{Fine Guidance Sensor/Two Gyro mode (F2G)}

The third and highest submode is the FGS/Two Gyro mode (F2G). Science is performed in F2G after FGS guide star acquisition and fine lock has been achieved on two FGSs. This process requires an initial simultaneous FHST and FGS visibility period during which body rates are held under tight control using an FHST until the first FGS has acquired a guide star and entered coarse track mode ${ }^{7}$. Once coarse track mode is entered the FGS effectively assumes rate control from the FHST further reducing body rates and jitter. Following this, the second FGS proceeds through its normal

\footnotetext{
7 The FGS units have a coarse tracking mode that is automatically entered after a spiral search pattern successfully finds a guide star. In coarse track mode the Instantaneous Field of View (IFOV) of the FGS follows a nutating scan pattern meant to track the guide star. Position information from the tracking is utilized to reduce body rates and establish an accurate position of the star for the transition to fine lock mode. The transition to fine lock mode involves a back off then walk down of the IFOV over the star's location that utilizes the interferometers to lock its position.
}

guide star acquisition process - guide star search, coarse track, and transition to fine lock. The transition to fine lock is made possible by the reduction in rate and jitter afforded by having the first FGS in coarse track.

\subsection{TGS Mode Transitions}

During a typical orbit each science observation period ends due to the occultation of the telescope boresight (and the FGSs). At this time an attempted transition down to $\mathrm{T} 2 \mathrm{G}$ mode is made by the Flight Software (FSW). Typically, however, there will be no FHST visibility at that time, thus forcing a further transition down to the $\mathrm{M} 2 \mathrm{G}$ mode. Once in M2G mode spacecraft attitude is held constant to reacquire the same target on the subsequent orbit, or a slew is performed to orient the boresight for a different target. In both cases, the large attitude errors that occur in $\mathrm{M} 2 \mathrm{G}$ mode must be eliminated by the two $\mathrm{OBADs}$ performed in $\mathrm{T} 2 \mathrm{G}$ mode prior to an attempt at the FGS guide star acquisition and the resumption of science imaging. The T2G mode thus plays a very important transitional role in attitude error reduction (from degrees to arcseconds) every orbit. To accomplish this effectively requires large periods (up to 30 minutes) of FHST visibility that adjoin the start of the FGS guide star acquisition (the start of F2G mode). See Figures 2 and 3 for depictions of the allowed transitions between the $\mathrm{M} 2 \mathrm{G}, \mathrm{T} 2 \mathrm{G}$, and F2G modes.

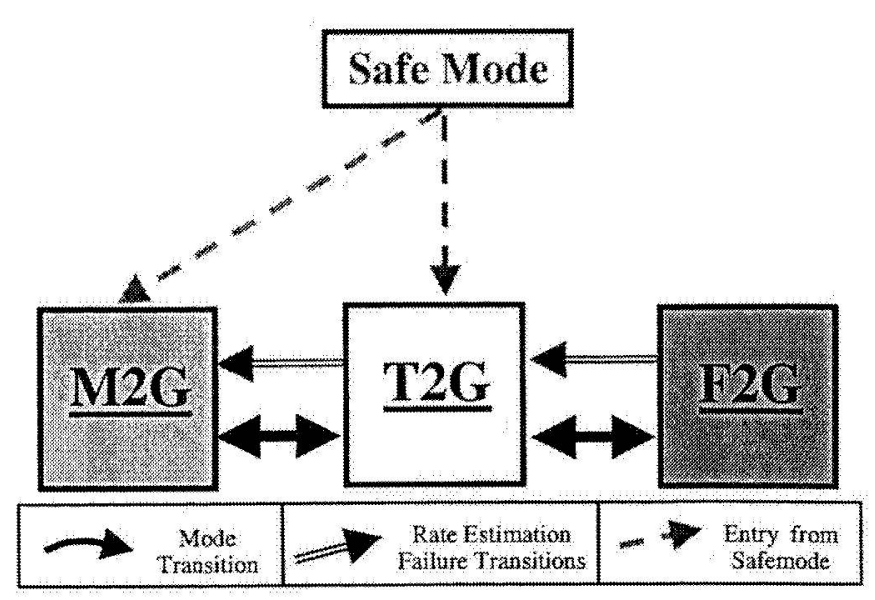

Figure 2. Allowed TGS submode transitions. 


\section{TWO GYRO DESIGN AND PERFORMANCE FOR THE HUBBLE SPACE TELESCOPE}
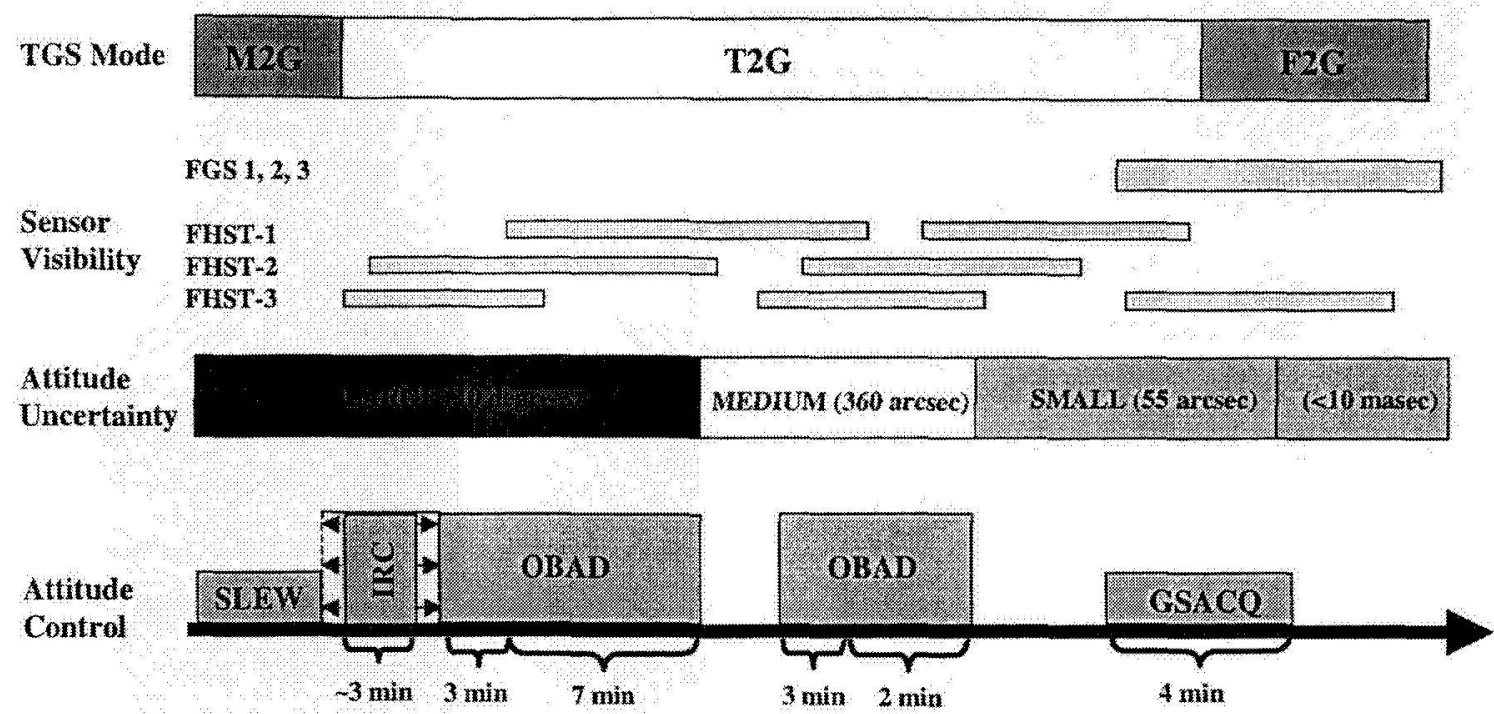

Figure 3. Typical TGS orbit scenario depicting TGS mode transitions, sensor visibility, and attitude uncertainty and control profiles. The Initial Rate Control (IRC) period, rate control period during and $-2 \mathrm{~min}$. prior to the Guide Star Acquisition (GSACQ), and the first 3 minutes of each OBAD require that no FHST handoff occur. The initial 3 minute period of an $O B A D$ is when star mapping operations are performed while the remaining time is used for the attitude correction maneuver. Durations shown are approximate.

\subsection{TGS Mode vs, 3 -Gyro Comparison}

A comparison between the current 3-gyro and TGS mode operations shows that, overall, total science observing time is preserved at approximately 55 minutes (per 96 minute orbit). The changes in the non-observing portion of the orbit are evident by examination of Figure 4 (page 6), which indicates the much larger portion of time during which FHST visibility is needed in T2G for performing the transition from $\mathrm{M} 2 \mathrm{G}$ to $\mathrm{F} 2 \mathrm{G}$ as described previously. In the 3-gyro mode, the rough equivalent of $\mathrm{T} 2 \mathrm{G}$ mode is the brief usage of the FHSTs for very small (no more than 300 arcseconds) attitude updates.

\section{SCIENCE PROGRAM IMPACTS}

Potential science program impacts under TGS operations concern science quality and net science yield. Quality is primarily driven by pointing jitter evidenced by the size of the Point Spread Function (PSF) of any calibrated target. The effect of increased jitter is a broadening of the PSF, reducing the Signal-to-Noise Ratio (SNR) of the image. Increased exposure times can be used to offset this and return the SNR to near previous levels. However, a commensurate reduction in total number of targets results since more time must be allotted to each target for increased exposure durations. During the early development stages of the TGS capability jitter levels were anticipated to be as high as 30 milliaresec $(60 \mathrm{sec}$, RMS, 10). On-orbit testing, however, demonstrated that jitter levels, even with the worstcase gyro pair, are nearly equivalent to 3-gyro mode (i.e. 8-10 milliarcsec vs. 3-4 milliarcsec for 3 -gyro mode). Figure 5 (page 6) depicts the PSF comparison between TGS and 3-gyro modes indicating that typical TGS PSFs are indistinguishable from 3-gyro mode.

Although jitter levels are higher than for 3-gyro mode they still nearly meet the 3-gyro jitter requirement of 7 milliarcsec, resulting in science quality that is indistinguishable from 3-gyro mode with no required increase in exposure time (Figure 6 on page 6 ).

With such high quality science capability available there are essentially no limitations present in the TGS mode with regard to use of image detectors, apertures, science instrument modes (e.g. 


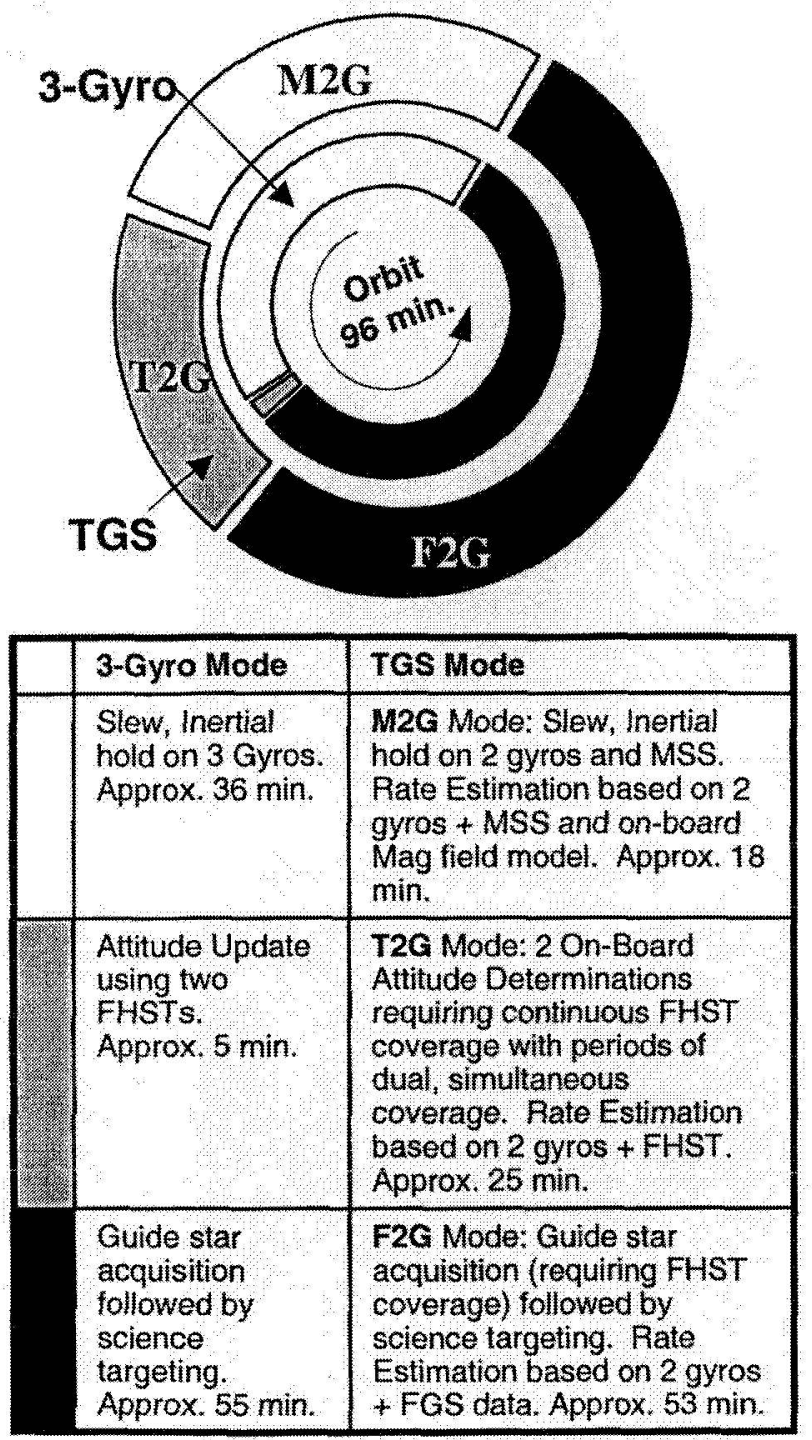

Figure 4. Summary comparison of TGS vs. 3-gyro modes. Note large duration difference in T2G mode vs. equivalent 3 -gyro period.

coronagraphy), etc. Three-gyro science quality can be achieved in TGS mode.

Science yield or quantity is driven by the attainable schedule efficiency (average number of orbits that can be scheduled for targeting over a unit of time) and the observation time achievable during a typical orbit. A primary factor in scheduling efficiency is the more difficult scheduling requirements imposed by the large amount of FHST visibility needed for T2G mode. Current science scheduling requires only brief use (on the order of 2-5 minutes) of the FHSTs to correct

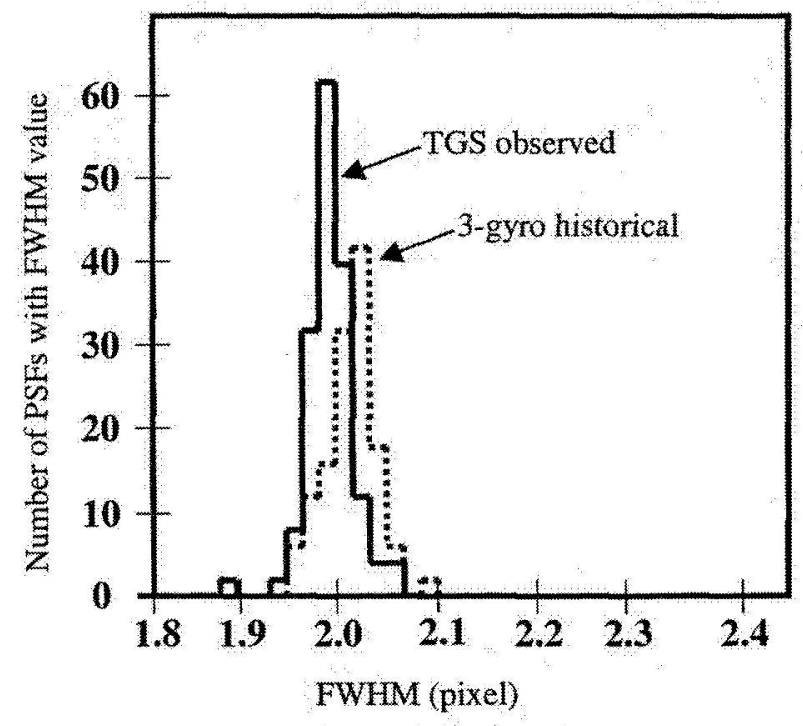

Figure 5. TGS vs. 3-gyro PSF comparison. Plots shown are PSF histograms, not actual PSF functions. TGS PSFS are, on average, slightly better than 3-gyro PSFs due to being measured just after a telescope focus adjustment. [Reprinted courtesy of Space Telescope Science Institute.]
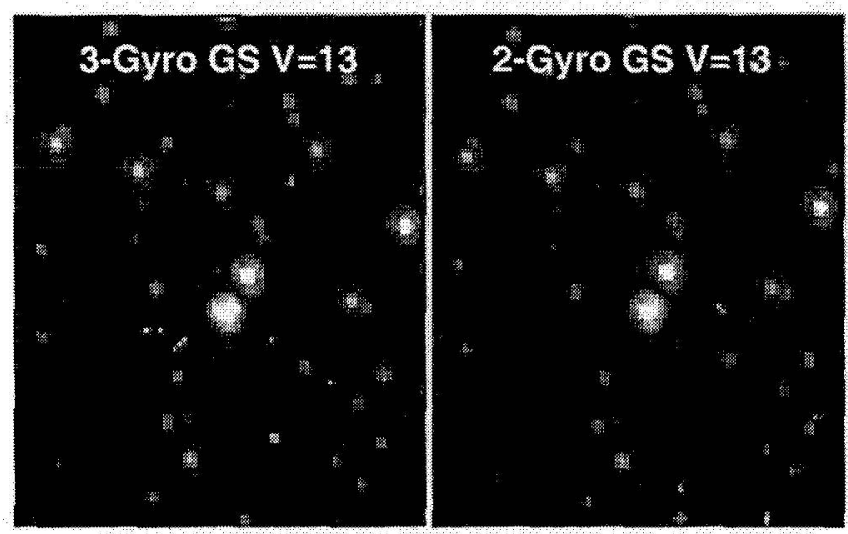

ACSHAC F555W - exposure time: 100 sec

Figure 6. TGS vs. 3-gyro image comparison for magnitude 13 stars. The dynamic range of the images have been logarithmically stretched to enhance tainter features. No discernable difference can be seen. [Reprinted courtesy of Space Telescope Science Institute.]

attitude errors built up during large slews. As discussed previously, TGS operations will require a period of up to 30 minutes of continuous FHST visibility among the 3 FHSTs (as a system) directly preceding the start of every science target. Additionally, two periods of dual and simultaneous FHST visibility are required to conduct attitude determinations and corrections. 


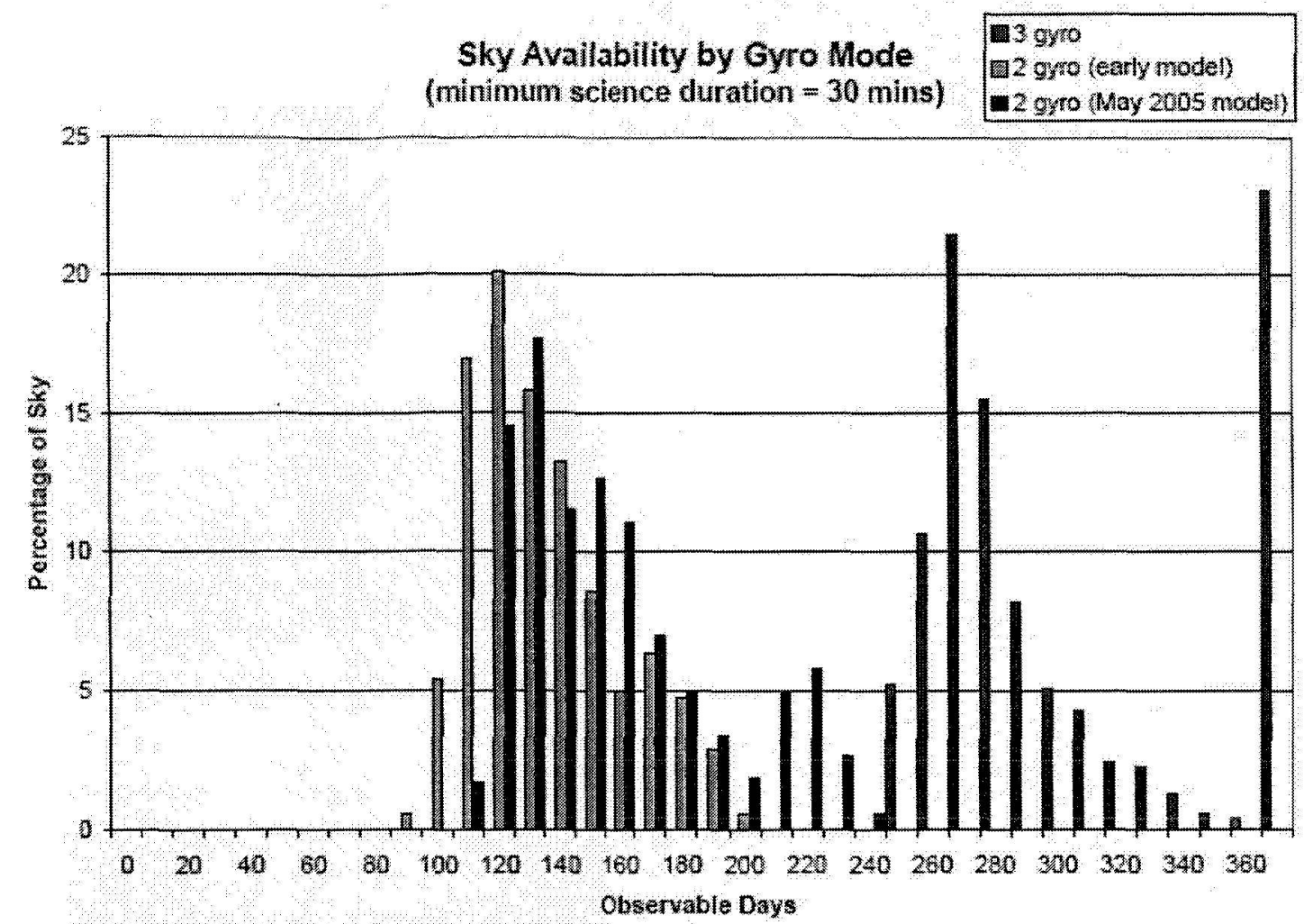

Figure 7. Percentage of sky vs. observable days comparison between TGS and 3 gyro modes: Improvements in the TGS early model were achieved by the adjustment of OBAD star mapping durations, maneuver times, and the attitude uncertainty following each $\mathrm{OB} A \mathrm{D}$. All areas of the sky are viewable in both TGS and 3-gyro modes but for a smaller duration in TGS mode. [Reprnted courtesy of Space Telescope Science Institute.]

Accommodating this requirement effectively reverses the current Observatory scheduling paradigm in which FHST usage is easily scheduled around science targeting, to the situation where science targets are selected based upon what areas of the sky can be targeted. (In turn driven by the fact that FHST visibility constraints can be met there.) The net effect will be that all areas of the sky can be targeted during TGS operations, but for a reduced number of days. Some areas are available only a few days a year while others will be available for weeks. Additionally, these periods of target area availability are most often not continuous, but occur as a series of segments spread over a larger period. The resulting effect is to render some of each week's orbits unusable because of the incompatibility of target locations and availability. Efforts have been completed to map the sky defining zones based upon right ascension and declination and their corresponding total viewing availability duration as well as the sensitivity of these durations to other factors such as differing levels of attitude uncertainty in each of the TGS submodes. Figure 7 depicts the integration over such a mapping to show the total number of days over which a specific percentage of sky can by targeted.

The other factor affecting sky availability is the increased sizes of the Sun, Earth and Lunar avoidance zones driven by the larger attitude errors inherent in TGS mode. For example, the telescope boresight Solar Avoidance Zone for 3-gyro mode is $50^{\circ}$. It must be increased to $60^{\circ}$ to account for the potential of a $10^{\circ}$ attitude error in $\mathrm{M} 2 \mathrm{G}$. The net effect of these two factors is reduced target availability over any given short-term period (i.e. over several weeks). However, all areas of the sky can still be viewed in TGS mode.

The other factor determining science quantity, the average observation time per orbit, mainly 


\section{TWO GYRO DESIGN AND PERFORMANCE FOR THE HUBBLE SPACE TELESCOPE}

influences the number of orbits that must be allocated to achieve the desired total image integration time. That is, the desired target time must be fit into the available observation time of an orbit. If the available observation time shrinks significantly due to TGS effects then it may be necessary to allocate more orbits to the target resulting in an overall reduction in science volume over the cycle.

In TGS mode, the average available observation time is affected by slightly longer guide star acquisition times and the need to perform a full acquisition each orbit. Longer FGS guide star acquisition durations are necessary due to the extra time needed to reduce body rates prior to the transition to fine lock control. A reduction in observation time results since guide star acquisitions must occur while the science target is visible and must be completed prior to the start of science imaging. Fortunately, the impact to observation time per orbit is very small since the extra time to perform acquisitions under TGS mode is on the order of 1 minute.

Full acquisitions must be performed each orbit, compared to the more rapid guide star reacquisition capability available in 3 -gyro mode for multi-orbit observations. This is due to the fact that a drop to M2G typically occurs every orbit forcing a full cycle of attitude error reduction (i.e. M2G-T2G$\mathrm{F} 2 \mathrm{G}$ transition) prior to the resumption of science imaging. However, this effect is also small, and when combined with the effect of slightly longer guide star acquisition times the result is only 1-2 minutes of reduced observation time per orbit. This does not materially affect science yield.

\section{TGS IMPLEMENTATION}

The implementation of the TGS mode began in the summer of 2003. To allow early FSW development and on orbit experience, the implementation was broken up into several builds that could be individually tested on-orbit. The algorithms for each submode were developed and provided separately by the Pointing Control
System group. This allowed for on-orbit testing of the On-board Attitude Determination (OBAD) FSW code in April of 2004, the T2G and M2G control modes in late 2004 and the full capability with F2G and science scheduling in February 2005. Numerous design reviews, peer reviews, code walk-throughs, and tests were held during that time. A final Operational Readiness Review (ORR) was then successfully held in April of 2005.

Following the ORR, it was agreed to proactively enter TGS to prolong the remaining gyro lifetime primarily driven by the excellent performance of the mode. To do this, however, required a reworking of science planning and scheduling. The observing schedule for HST is laid out in annual proposal cycles at the Space Telescope Science Institute (STScI). Observers design their proposals for a desired scenario including requested orbits, attitude, instrument configurations, integration times, etc based on observing guidelines. STScI prepared a new set of guidelines for TGS observing and provided it in their call for proposals for the next science cycle - Cycle 14 (August 2005 August 2006). This allowed observers to optimize their orbits based on TGS scenarios including TGS sky availability restrictions. Alternatively, to redesign observing proposals that were already in the pipeline (i.e. Cycle 13 proposals already designed for 3-gyro operations) would have been a large effort. A transition date of the middle of August 2005 was selected based upon having enough of the Cycle 14 proposals in the pipeline ready to execute and only a few remaining Cycle 13 proposals that would need to be reworked into the TGS observing format.

\section{EXPECTED SCIENCE GAINS}

The decision to intentionally enter TGS mode to preserve gyro lifetime for later use required analysis to understand the potential benefit to overall science productivity and yield. Observatory scheduling efficiency is reduced in TGS mode (for the reasons discussed above) and the ability for F2G to maintain fine lock throughout 
an observation is not as robust to anomalies. The major contributors to scheduling inefficiencies are the need to avoid poor magnetic field alignments during spacecraft slewing (in M2G mode), scheduling around South Atlantic Anomaly (SAA) impacted orbits, and reduced sky availability for targeting due to the needed FHST visibility. observing, an average effective scheduling rate of 80 prime science orbits a week is realized ${ }^{8}$. The total number of orbits was predicted for two cases assuming a starting point of August 2005 (the beginning of the next science cycle), and that a gyro failure would occur at a time corresponding to the $50 \%$ probability point taken from the gyro

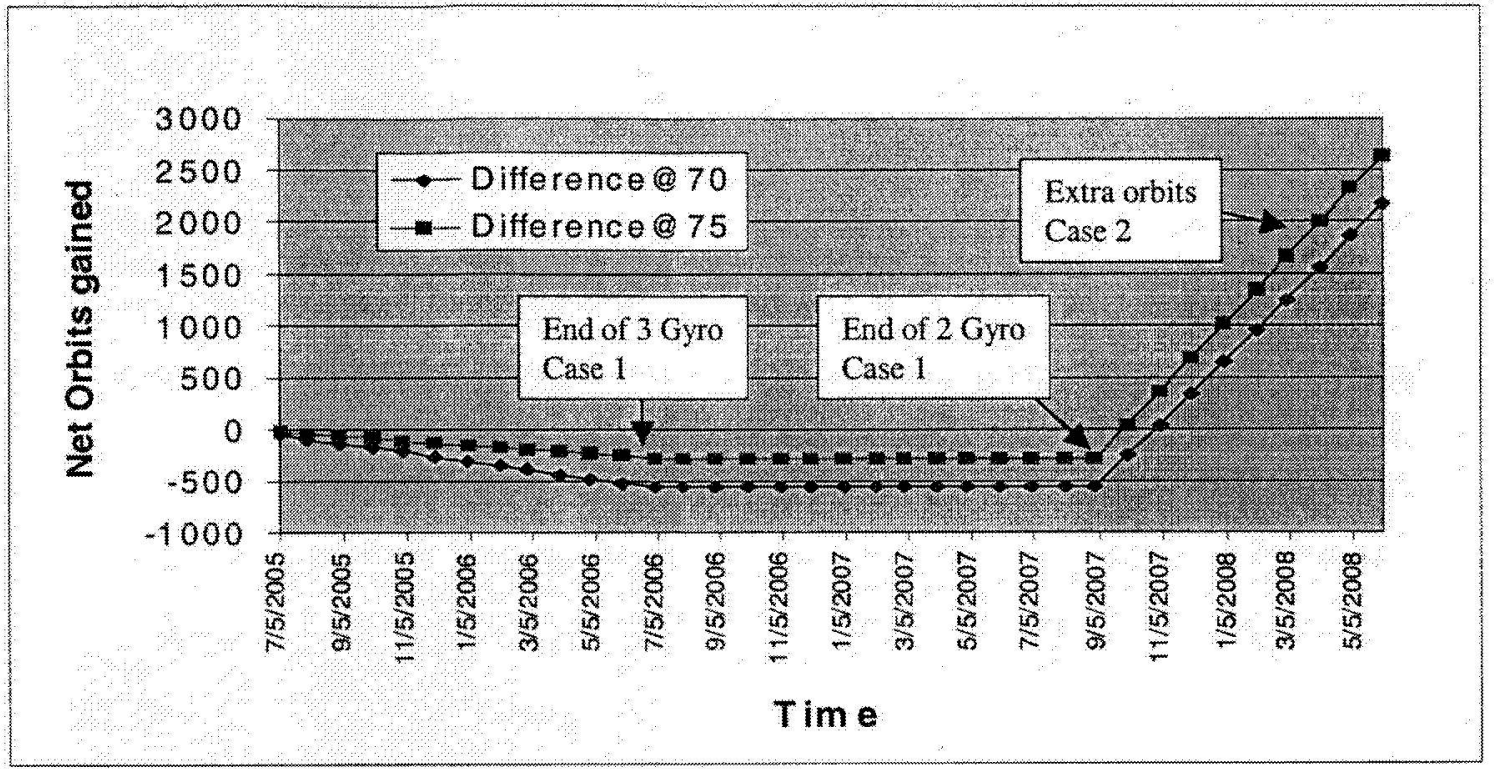

Figure 8. Net orbits gained vs. time for a proactive TGS mode use for levels of scheduling rate of 70 and 75 prime science orbits/week. Net orbits gained is defined as the difference in total orbits between proactive use of TGS mode at the start of the Cycle 14 science program (Case 2) compared to use of TGS mode only after 3 -gyro mode is no longer viable (Case 1). The plot starting point is one month earlier than the planned beginning of science Cycle 14. [Reprinted courtesy of Space Telescope Science Institute.]

Therefore, there are on average fewer orbits of prime science per week operating in TGS mode. On the other hand, based on TGS mode jitter performance, there is not a need to allocate additional orbits to compensate for a reduced signal to noise ratio.

By proactively entering TGS mode NASA expects to continue the HST science program significantly longer compared to either having only 3-gyro mode available, or using the TGS capability only after operating for as long as possible with 3 -gyro mode. Understanding the increase depends upon determining where the potential gain in orbits (by operating longer) justifies the certain near-term loss of orbits by operating in TGS mode, when 3 gyro mode would still be available. In 3-gyro reliability model discussed earlier. The first case (use of TGS as a contingency mode in which operations with three gyros are continued as long as possible followed by TGS mode utilization Case 1) resulted in one year of three gyro operation and 14 months in TGS mode. In this case an unplanned transition from three to two gyro operations occurs resulting in temporary

\footnotetext{
${ }^{8}$ Prime science orbits are defined as the allocation given to General Observers (GO), Space Telescope Science Institute Director's Discretionary (DD) time, Guaranteed Observer (GTO) time, Treasury Programs, Calibration programs, etc. Approximately 80 prime orbits out of the 105 physical orbits/week are schedulable. The South Atlantic Anomaly (SAA) impacted orbits ( $-16 \%$ of the time is spent in the SAA), breaks many orbits up into pieces that can still be used for prime science. However, two or more SAA impacted orbits are often required to implement a single prime orbit.
} 


\section{TWO GYRO DESIGN AND PERFORMANCE FOR THE HUBBLE SPACE TELESCOPE}

inefficiency in the transition estimated at a loss of 100 orbits. Proactive entry into TGS mode (Case 2) resulted in 35 months in TGS mode over the entire period ${ }^{9}$.

Using this approach and accounting for differences in the scheduling efficiency and target success rates between 3-gyro and TGS modes, the total net orbit gain for a proactive TGS entry as function of time was calculated and is plotted in Figure 8 (page 9). The plot has three distinct zones corresponding to the changes in the operating mode of Case 1. The initial segment is declining due to the fact that in this period 3 -gyro mode would still be viable and result in a higher effective scheduling rate than TGS mode. The result is a negative net gain since the comparison is how well proactive use of TGS mode (Case 2) increases science orbits over use as a pure contingency mode (Case 1). The intermediate segment is flat since both cases result in TGS operations and thus produce an identical number of science orbits. The last segment is steeply increasing and begins where Case 1 ends. In this period, use of TGS mode proactively is still viable for some time and all science orbits scheduled are pure gain.

Consequently, if HST does not incur any other failure that impacts the ability to perform science then the end of 2007 marks the breakeven point after which continued operations provided by early proactive utilization of the TGS mode will increase the science productivity of the Observatory beyond the strategy as a purely contingency mode (i.e. Case 1). The curves shown are for effective TGS scheduling rates of 70 and 75 orbits/week ${ }^{10}$.

\footnotetext{
${ }^{9}$ The results cited in this section are based upon an analysis using a different gyro pair combination than used in section 2 (Life Extension Potential). Additionally, the estimates of life extension potential noted in section 2 are measured from the end of 3-gyro operations, assuming 3-gyro mode is used as long as possible. Both analyses assume a proactive TGS entry.

10 The TGS effective scheduling rate is lower than the -80 orbits/week realized in 3-gyro mode due to the affect of reduced target availability. The net observing rate is still lower and takes into account additional orbits lost due to
}

Scheduling studies done with the Cycle14 science program predicts a TGS rate of about 72 orbits/week (the breakeven point for proactive use of TGS mode is somewhat insensitive to effective scheduling rates over a small range). Given the superb quality of TGS mode science, the expected overall gain in science, and the ability to avoid a science hiatus, NASA has decided to go forward with the proactive entry into TGS mode.

\section{CONCLUSION}

The TGS performance exceeded Project expectations allowing NASA to use it proactively to extend the productive science mission of HST at the same unmatched level of science quality for an estimated 2 years. It was developed and implemented with minimal interruption to the existing HST science program.

Of course, the result of such success is the desire to extend the science lifetime of the Observatory even further by the development of a One Gyro Science (OGS) mode based on the TGS concepts. Initial studies give us a high level of confidence that a OGS mode is feasible. The fine pointing requirements that make HST unique as a world class observatory are met using the FGS units during science observing. The success in the TGS mode is due to using a combination of sensors to compensate for the lack of gyro information. Studies show that the MSS and FHST units can be used to compensate for two missing gyro axes as they did for one axis. The key development will be the robustness of the design to manage anomalies such as the FHST detecting non-stellar objects when there are fewer sensor inputs to use for data integrity checks,

problems with the science imaging that are present equally in 3 -gyro and TGS modes $(-2.5 \%)$, and the fact that TGS mode is less robust to guide star anomalies. All of these affects are accounted for in the plots of Fig. 8 . 